\title{
Coordinated Beamforming with Altruistic Precoding and User Selection for MU-MIMO System
}

\author{
Gaofeng Cui,, ${ }^{1,2}$ Yanjie Dong, ${ }^{2,3}$ Weidong Wang, ${ }^{1,2}$ and Yinghai Zhang ${ }^{1,2}$ \\ ${ }^{1}$ Key Laboratory of Universal Wireless Communications, Ministry of Education, Beijing University of Posts and Telecommunications, \\ Beijing 100876, China \\ ${ }^{2}$ Information \& Electronics Technology Lab, School of Electronic Engineering, Beijing University of Posts and Telecommunications, \\ Beijing 100876, China \\ ${ }^{3}$ China Mobile Group Design Institute Co., Ltd., Beijing 100080, China
}

Correspondence should be addressed to Gaofeng Cui; cuigaofeng@bupt.edu.cn

Received 14 January 2014; Accepted 2 June 2014; Published 18 June 2014

Academic Editor: Yulong Zou

Copyright (C) 2014 Gaofeng Cui et al. This is an open access article distributed under the Creative Commons Attribution License, which permits unrestricted use, distribution, and reproduction in any medium, provided the original work is properly cited.

Other cell interference (OCI) degrades the achievable capacity of downlink multiuser multiple-input multiple-output (MU-MIMO) systems seriously. Among OCI mitigation schemes, methods that sacrifice $\xi$ degrees of freedom to nullify the OCI have been proven to be helpful to improve the cell edge throughput. However, since interference nulling schemes can only improve the signal to interference plus noise ratio (SINR) of $\xi$ users, they are not optimal in terms of average cell throughput, especially for low to medium OCI levels. We explore the question whether it is better to improve the SINR of every user in other cells rather than benefit $\xi$ users. An altruistic precoding method to minimize the sum of generated interference for all of the other cell users is proposed with $\xi$ degrees of freedom being sacrificed. With the altruistic precoding method, we deduce the lower bound on the capacity and solve the multicell user selection problem with a local optimal solution in which only eigenvalues of interfering channels are needed to be shared. Simulation results demonstrate that the proposed method outperforms the existing algorithms at any OCI level. Furthermore, we also analyze the best choice of degrees of freedom used to mitigate OCI through simulation.

\section{Introduction}

With the requirement of high spectral efficiency, multiuser multiple-input multiple-output (MU-MIMO) needs to be implemented for IMT-advanced systems with dense frequency allocation. However, since every antenna in MUMIMO systems can be seen as an interfering source, interuser interference (IUI) and other-cell interference (OCI) are both needed to be tackled carefully [1]. Most of prior works, such as dirty paper coding (DPC) [2], block diagonalization (BD) [3], focus on eliminating the IUI thoroughly for a single cell. However, they do not consider the negative effects of OCI which can degrade the achievable capacity of cell edge user so much that the cell edge users' quality of service (QoS) cannot be guaranteed. Furthermore, the methods used in single cell scenario cannot be simply extended to multicell environment. In order to provide continuous service all over the coverage area, OCI management is crucial for future wireless cellular systems, especially for the MU-MIMO systems.

In [4], authors analyze the effects of OCI in MUMIMO network and compared several techniques that can be used to mitigate the OCI. Recently, coordinated multipoint (CoMP) is proposed as an effective way to increase the cell edge user throughput for MU-MIMO systems and has been adopted as one of the key techniques for $4 \mathrm{G}$ cellular systems, such as LTE-advanced [5] and IEEE $802.16 \mathrm{~m}$. Generally, downlink CoMP can be divided into two categories: joint processing/transmission (JPT) and coordinated scheduling/beamforming (CSB). For JPT, the data to UE is transmitted from multiple base stations (BSs) jointly, and the OCI seen by single BS processing is transferred to the desired signals. Although JPT can eliminate the OCI thoroughly in theory, its achievable performance gain relies on vast signaling overhead brought by sharing data and channel state information (CSI). While for CSB, the data to UE is 
transmitted from a single BS with no or partial CSI sharing, and the OCI is mitigated through coordinated scheduling and beamforming decisions of multiple BSs.

1.1. Related Work. With JPT, the cooperative BSs form a virtual MIMO broadcast channel in the downlink, and the cell edge throughput mainly benefits from designing the precoding matrices cooperatively with data and CSI sharing across multiple BSs. It has been proven that DPC is an optimal precoding method for broadcast channel [6]. However, DPC is hard to be deployed due to its high complexity. In [7], several practical joint transmission schemes with per BS power constraints are studied and advantages of cooperative processing in downlink multicell MU-MIMO networks are testified. Authors in [8] also analyze the maximum achievable common rate with cooperative transmission and indicate the enormous gain over single BS processing. In [9], BD is adopted with multicell joint processing to maximize the weighted sum rate of all UEs. However, all of these studies assume global coordination with full CSI and data sharing to eliminate the OCI thoroughly. In practice, since the backhaul capacity between BSs is limited and the time and phase cannot be precisely synchronized, the performance of JPT is seriously affected by the size of cooperating sets and signaling overhead brought by CSI and data sharing. The performance gain of downlink cooperative processing with limited backhaul capacity is discussed in $[10,11]$. In [10], the authors split the user data to the shared data and nonshared data and point out how much of the user data is worth sharing with finite backhaul capacity. Authors in $[12,13]$ investigate the achievable capacity of MU-MIMO with imperfect CSI, such as estimation error, feedback delay, and quantized error. Different from the above train of thought, cluster-based coordination is an effective way to reduce the complexity and maintain the throughput gain of joint processing simultaneously. In [14], a three-cell cooperating set is investigated with zero-forcing beamforming (ZFBF) in downlink, but the intercluster interference is not considered, which leads to the poor performance in the edge area of each cluster. Clustered MIMO with intercluster coordination is studied in [15-18] to explore the benefits of joint transmission while limiting the CSI feedback. In [15], the intercluster interference seen by edge users is precanceled at neighboring clusters with interference nulling precoding techniques, whereas this method can only be effective when there are sufficient degrees of freedom at neighboring clusters.

For another kind of downlink CoMP which is named as $\mathrm{CSB}$, the cell edge throughput gain mainly comes from scheduling and beamforming cooperatively among multiple BSs. Compared with JPT, since CSB does not need to share data via backhaul links, it is more practical to be implemented. In [19], the capacity gain that can be achieved from intercell scheduling is analyzed. Several downlink beamforming methods are investigated in [20-22] for MUMIMO networks. In [20], the interference-aware precoder is adopted at the transmitter and an interference whitening filter is implemented at receiver to mitigate the OCI from adjacent cells. In [22], a joint beamforming and power allocation method is proposed to maximize the worst-user signal to interference and noise ratio (SINR). Different from the above methods that mitigate OCI passively, the researchers are also interested in the concept of suppressing the interference generated to other cells users proactively. Distributed precoding methods based on signal to caused interference ratio (SCIR) and virtual SINR (VSINR) are given for MISO systems in [23-25], respectively. In [26], precoders based on signal to leakage plus noise (SLNR) are derived for users with both single and multiple data streams. In order to tackle both intracell and other-cell interference, the authors in [27] proposed a nonlinear distributed precoder with DPC. In [27], the other-cell interference is limited by maximizing cellbased SLNR followed with nullifying intracell interference by DPC. In [28], the authors maximize the weighted SLNR to guarantee the weak users' performance. However, all of the above studies do not consider consuming some spatial degrees of freedom to cancel OCI. In [29], the authors explore the question whether it is better to do OCI cancellation (OCIC) than simply single-cell beamforming and propose an adaptive transmission scheme. In [30], an approach for OCI suppression was proposed for multiusers case with user selection by using partial degrees of freedom to support its users and utilizing the residual $\xi$ degrees of freedom to nullify the OCI. Although the OCI cancellation schemes can bring throughput gain when the OCI level at the cell edge is high, they are not always optimal in terms of average cell throughput with the SINR of $\xi$ users being improved merely, especially for low to medium OCI levels. This is mainly because them following: (1) since OCI cancellation can only improve the SINR of $\xi$ users, the throughput gain brought by OCI cancellation mainly depends on the OCI levels of beneficial users. When the OCI level is low, the throughput gain cannot compensate the throughput loss due to the sacrifice of degrees of freedom; (2) since the number of antennas implemented at each BS is limited, the number of interfered users by a given BS is generally greater than the degrees of freedom that can be used for OCI cancellation. OCI cancellation for $\xi$ users cannot guarantee the minimization of the sum of generated interference bore by all of interfered users.

1.2. Contribution. In this paper, we consider a multicell network with CSB being adopted for interference mitigation. With partial CSI and no data sharing, we investigate the question whether it is better to improve the SINR of every interfered user rather than favor $\xi$ users. Suppose that $N$ antennas are implemented at each BS and $\xi$ degrees of freedom can be used to mitigate OCI. The main contributions of this paper are summarized as follows.

(i) Altruistic Precoding Based on Interference Assignment. Unlike prior works that nullify the OCI for $\xi$ users thoroughly, we propose a precoding method that can assign no IUI for $N-\xi$ users and limited OCI for the other cell users by using residual $\xi$ degrees of freedom to minimize the sum of generated interference. Compared with the scheme that nullifies OCI for $\xi$ users, the proposed method can distribute the benefits of OCI mitigation to all of the interfered 
(1) Stage $n=0$ : Set all users inactive. For $t$ th TTI, let the selected user set $\Omega_{i}(t)=\emptyset$, unselected user set $\Psi_{i}(t)=\left\{1,2, \ldots, \bar{K}_{i}(t)\right\}$.

The number of selected users served by other BS is assumed as an integer $K_{j}$, and $0 \leq K_{j} \leq N$.

(2) Stage $n=1$ : For each BS $i$, find the best user $k$ with largest lower bound on the capacity

$$
\bar{C}_{k, i}(t)=\max _{k=1,2, \ldots, \bar{K}_{i}(t)} \log _{2} \frac{\left\|H_{k, i}\right\|_{F}^{2}}{\sigma_{n}^{2}+\sum_{j \neq 1}^{J} K_{j} \lambda_{1}\left(H_{k, j} H_{k, j}^{H}\right)}
$$

Let $\Omega_{i}(t)=\Omega_{i}(t)+\{k\}, \Psi_{i}(t)=\Psi_{i}(t)-\{k\}$.

(3) Stage $n=n+1$ :

(a) Temporarily activate the user $k$ in candidate user set $\Psi_{i}(t)$, and calculate the lower bound on the capacity $\bar{C}_{k, i}(t)$ shown in (24).

(b) For each BS $i$, find the user $k$ with the maximum sum capacity,

$$
(k, i)=\arg \max _{k \in \Psi_{i}(t)} \bar{C}_{k, i}
$$

(c) For each BS $i$, let $\Omega_{i}(t)=\Omega_{i}(t)+\{k\}, \Psi_{i}(t)=\Psi_{i}(t)-\{k\}$. If $n \leq N-\xi$, return to (3). Else, quit the algorithm.

Algorithm 1: Decentralized user selection with interference assignment.

users and improve the SINR of every user in adjacent cells. Simulation result demonstrates that 5\% CDF of SINR with $\xi=1$ for the proposed precoding method is $5 \mathrm{~dB}$ higher than that for OCI cancellation schemes. Moreover, since the proposed method can be implemented with partial CSI and no data sharing, it is more practical for realistic networks that have limited backhaul capacity.

(ii) User Selection Based on the Lower Bound on the Capacity. With the precoding method, the lower bound on the capacity for a single user is derived with the maximum eigenvalue of interfering channels being shared merely. Thus, the global optimal problem of user selection for multicell is reformulated to a local optimal problem that can be tackled by each BS without centralized processing entities. With $N=$ 4 and $\xi=1$, it is shown from simulation results that the proposed user selection method, named as decentralized user selection with interference assignment (DUSIA), can increase the cell edge throughput and average cell throughput of user selection with interference nulling scheme up to $70 \%$ and $20 \%$, respectively (Algorithm 1). In addition, DUSIA can achieve $99 \%$ average cell throughput of centralized user selection scheme with full CSI sharing and centralized processing entities.

(iii) Best Choice of $\xi$. Since using $\xi$ degrees of freedom to mitigate OCI can lead the number of active user decreasing to $N-\xi$, there is a tradeoff between OCI mitigation and achievable throughput. In this paper, we find that the larger the $\xi$ can get, the more cell edge throughput can be achieved. While for the term of the average cell throughput, there is always a turning point with the increasement of $\xi$.

1.3. Organization. The remainder of this paper is organized as follows. The system model is listed in Section 2. In Section 3, an altruistic precoding based on interference assignment is proposed by suppressing the OCI for other cell users.
Section 4 formulates the problem of user selection under the scenario of multicell MU-MIMO and describes a decentralized mechanism to resolve the problem. System level simulation and analysis are given in Section 5 to find the best choice of $\xi$ in different scenarios. And Section 6 concludes the paper.

Throughout the paper, we will use the following notations. Let $A^{H},|A|,\|A\|_{F}, A^{-1}$, and $\operatorname{tr}(A)$ denote the complex conjugate transpose, determinant, Frobenius norm, inverse, and trace of the matrix $A$, respectively. $\lceil\cdot\rceil$ stands for the ceiling operations, and $E\{\cdot\}$ denotes the expectation operation.

\section{System Model}

Consider a downlink multicell MU-MIMO network with $N$ antennas implemented at each BS. Each user equipment (UE) is assumed to have a single antenna. For the scenario of multiple antennas being implemented at each UE, the proposed scheme can also be applied with each antenna being seen as a single user. Before describing the proposed scheme, we make several assumptions as follows.

Assumption 1. Each BS is aware of the perfect CSI for the channel between the BS and its serving users, as well as interfering users. As shown in Figure 1, BS1 is aware of the perfect CSI for the channel between BS1 and UE1, as well as the channel between BS1 and UE4. For each BS, CSI of its serving users is used to cancel the IUI, while the CSI between users in neighboring cells and the BS is used to mitigate the generated interference to the other cell users. In time division duplexing (TDD) systems, each BS can obtain the downlink CSI of each user by uplink channel estimation, while, in frequency division duplexing (FDD) systems, the downlink CSI can be obtained by feedback from users. Although the imperfect feedback and channel estimation can have large impacts on system capacity, these issues are beyond the scope of this paper.

Assumption 2. Different BSs can exchange specific information except for user data through the backhaul. In the 


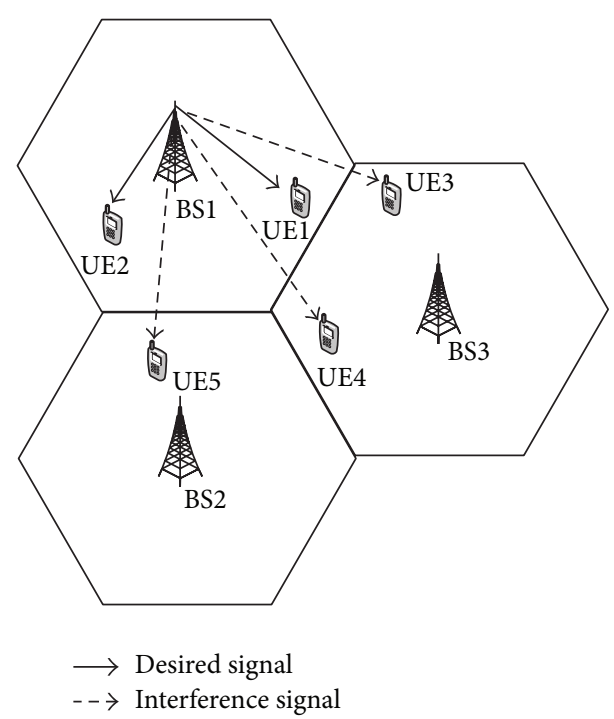

FIGURE 1: MU-MIMO system with generated interference.

proposed scheme, since the data to a user is transmitted from a single BS, data sharing is not needed. However, in order to do coordinated beamforming, some specific information, such as indices of active users and eigenvalues of interfering channels, needs to be exchanged through backhaul. In LTE/LTE-advanced systems, these all can be exchanged over $\mathrm{X} 2$ interface [31].

With the above assumptions, suppose $\xi$ degrees of freedom can be used to mitigate OCI, for a network consisting of $K_{i}$ users served by each BS $i$, the postprocessing receive signal $r_{k, i}$ for user $k$ served by BS $i$ can be represented as

$$
\begin{aligned}
r_{k, i}= & H_{k, i} W_{k, i} x_{k, i}+\sum_{l=1, l \neq k}^{K_{i}} H_{k, i} W_{l, i} x_{l, i} \\
& +\sum_{j \neq i}^{J} \sum_{\bar{k}=1}^{K_{j}} H_{k, j} W_{\bar{k}, j} x_{\bar{k}, j}+n_{k, i} .
\end{aligned}
$$

In (1), $H_{k, i}$ is a $1 \times N$ matrix that denotes the channel between the BS $i$ and user $k$. The transmit signal of user $k$ is denoted as $x_{k, i}$ with an average power constraint $E\left\{x_{k, i} x_{k, i}^{H}\right\}=1 . W_{k, i}$ is a precoding matrix for user $k$. $J$ denotes the number of BSs in the network. The second and third items in the righthand side of (1) denote IUI and OCI, respectively. $n_{k, i}$ is the additive complex Gaussian noise vector with zero mean and $\sigma_{n}^{2}$ variance.

\section{Altruistic Precoding Based on Interference Assignment}

In this paper, we focus on the generated interference by the BS to the other cell users. Thus, the explicit information of interference from other cells, such as the CSI of interfering channels and precoding matrices, is not needed to be shared via the backhaul link whose capacity is limited. Generally, the transmited data of a given user can cause IUI and
OCI simultaneously. In order to mitigate the generated interference proactively, we need to answer the following two questions:

(i) how to assign IUI among users served by the same cell?

(ii) how to assign OCI generated to users in adjacent cells?

For IUI, since it comes from the serving BS, the strength of desired signal and IUI signal received by a given user is almost the same. If IUI cannot be nullified, it would cause the user's achievable capacity to be seriously degraded, especially for the cell-center users near the serving BS. While for OCI, since interference nulling can only improve the SINR of limited number of users, it is not always optimal to sacrifice $\xi$ degrees of freedom to nullify the generated interference for $\xi$ users. Therefore, we intend to assign the generated interference to satisfy the following requirements:

(i) the IUI assigned for users served by the same cell can be nullified;

(ii) the sum of OCI assigned for all of the users in adjacent cells rather than that of $\xi$ users can be minimized.

As is shown in Figure 1, interference nulling can only eliminate the generated interference for one user (UE3) with $\xi=1$. However, we intend to minimize the sum of generated interference for all of users (UE3, 4, 5) in adjacent cells.

In order to satisfy the requirements listed above, we first obtain a precoding matrix that can nullify the IUI, so that the MU-MIMO channel can be divided into several parallel SU-MIMO channels. And then, a filter matrix is implemented to mitigate the generated interference by minimizing every user's aggregated leakage plus noise (LN). Thus, we can rewrite the precoding matrix $W_{k, i}$ as a product of two matrices shown as

$$
W_{k, i}=Q_{k, i} G_{k, i}
$$

where $Q_{k, i}$ is used to nullify IUI and $G_{k, i}$ is used to mitigate the generated interference to other users in neighboring cells. To nullify IUI, $Q_{k, i}$ should satisfy the following constraints described as

$$
H_{k, i} Q_{l, i}=0 \quad \forall k \neq l .
$$

In order to satisfy (3), $Q_{k, i}$ should lie in the null space of $\bar{H}_{k, i}$ that can be expressed as

$$
\bar{H}_{k, i}=\left[H_{1, i}^{H}, H_{2, i}^{H}, \ldots, H_{k-1, i}^{H}, H_{k+1, i}^{H}, \ldots, H_{N-\xi-1, i}^{H}\right]^{H} .
$$

In (4), since $\xi$ degrees of freedom are used to mitigate OCI, only $N-\xi$ users can be supported with $N$ transmit antennas. Denote the singular value decomposition (SVD) of $\bar{H}_{k, i}$ as $\bar{H}_{k, i}=U_{k, i}\left[\Lambda_{k, i} 0\right]\left[V_{k, i}^{(1)} V_{k, i}^{(0)}\right]^{H}$, where $V_{k, i}^{(0)}$ is a $N \times(\xi+1)$ matrix and denotes the null space of $\bar{H}_{k, i}$. Thus, the precoding matrix $Q_{k, i}$ can be expressed as $V_{k, i}^{(0)}$, and the equivalent 
channel matrix for user $k$ can be expressed as $H_{k, i} V_{k, i}^{(0)}$. If (3) is satisfied, (1) can be rewritten as

$$
r_{k, i}=H_{k, i} Q_{k, i} G_{k, i} x_{k, i}+\sum_{j \neq i}^{J} \sum_{\bar{k}=1}^{N-\xi} H_{k, j} Q_{\bar{k}, j} G_{\bar{k}, j} x_{\bar{k}, j}+n_{k, i} .
$$

As it is shown in (5), when the IUI is nullified by $Q$, the OCI is mainly affected by $G$. In this paper, we intend to mitigate OCI by minimizing each user's aggregated LN. Let the equivalent desired channel matrix and interfering channel matrix be as $\widetilde{H}_{k, i}=H_{k, i} V_{k, i}^{(0)}$ and $\widetilde{H}_{\bar{k}, i}=H_{\bar{k}, i} V_{k, i}^{(0)}$, respectively. The aggregated LN of user $k$ can be expressed as [26]

$$
\mathrm{LN}_{k, i}=\sigma_{n}^{2}+E\left[\sum_{\bar{k} \in \Omega_{i}} x_{k, i}^{H} G_{k, i}^{H} \widetilde{H}_{\bar{k}, i}^{H} \widetilde{H}_{\bar{k}, i} G_{k, i} x_{k, i}\right],
$$

where $\Omega_{i}$ is the user set interfered by BS $i$. Here, $\operatorname{LN}_{k, i}$ represents the aggregated leakage signal and noise power of user $k$, and the leakage signal denotes the interference caused by the precoding matrix of a specific user on other cell users. Since $x_{k, i}$ is a $1 \times 1$ vector, (6) can be expressed as

$$
\begin{aligned}
\mathrm{LN}_{k, i} & =\sigma_{n}^{2}+E\left[\sum_{\bar{k} \in \Omega_{i}} x_{k, i}^{H} G_{k, i}^{H} \widetilde{H}_{\bar{k}, i}^{H} \widetilde{H}_{\bar{k}, i} G_{k, i} x_{k, i}\right] \\
& =\sigma_{n}^{2}+E\left[\sum_{\bar{k} \in \Omega_{i}} \operatorname{tr}\left(x_{k, i}^{H} G_{k, i}^{H} \widetilde{H}_{\bar{k}, i}^{H} \widetilde{H}_{\bar{k}, i} G_{k, i} x_{k, i}\right)\right] .
\end{aligned}
$$

According to $\operatorname{tr}(A B)=\operatorname{tr}(B A)$ and $E\left\{x_{k, i} x_{k, i}^{H}\right\}=1$, (7) can be rewritten as

$$
\begin{aligned}
\mathrm{LN}_{k, i} & =\sigma_{n}^{2}+E\left[\sum_{\bar{k} \in \Omega_{i}} \operatorname{tr}\left(x_{k, i}^{H} G_{k, i}^{H} \widetilde{H}_{\bar{k}, i}^{H} \widetilde{H}_{\bar{k}, i} G_{k, i} x_{k, i}\right)\right] \\
& =\sigma_{n}^{2}+\sum_{\bar{k} \in \Omega_{i}} \operatorname{tr}\left(G_{k, i}^{H} \widetilde{H}_{\bar{k}, i}^{H} \widetilde{H}_{\bar{k}, i} G_{k, i}\right) .
\end{aligned}
$$

When (8) is used as a cost function, $G_{k, i}$ that can minimize aggregated LN of a user is benefit to the system capacity. We can express $G_{k, i}$ corresponding to the minimum LN as

$$
G_{k, i} \propto \arg \min _{G_{k, i} \in C^{(\xi+1) \times 1}} \mathrm{LN}_{k, i} .
$$

Since $\mathrm{LN}_{k, i}$ is a scalar and $G_{k, i}$ is normalized as $\operatorname{tr}\left(G_{k, i}^{H} G_{k, i}\right)=$ $1, G_{k, i}$ also can be derived as

$$
\begin{gathered}
G_{k, i} \propto \arg \max _{G_{k, i} \in C^{(\xi+1) \times 1}}\left(\sigma_{n}^{2}+\sum_{\bar{k} \in \Omega_{i}} \operatorname{tr}\left(G_{k, i}^{H} \widetilde{H}_{\bar{k}, i}^{H} \widetilde{H}_{\bar{k}, i} G_{k, i}\right)\right)^{-1} \\
\propto \arg \max _{G_{k, i} \in C^{(\xi+1) \times 1}} \frac{\operatorname{tr}\left(G_{k, i}^{H} G_{k, i}\right)}{\sigma_{n}^{2}+\sum_{\bar{k} \in \Omega_{i}} \operatorname{tr}\left(G_{k, i}^{H} \widetilde{H}_{\bar{k}, i}^{H} \widetilde{H}_{\bar{k}, i} G_{k, i}\right)} .
\end{gathered}
$$

Therefore, $G_{k, i}$ can be derived as [26]

$$
G_{k, i} \propto \max _{G_{k, i} \in C^{(\xi+1) \times 1}} \text { eigenvector }\left(\left(\sigma_{n}^{2} I_{\xi+1}+\widehat{H}_{\bar{k}, i}^{H} \widehat{H}_{\bar{k}, i}\right)^{-1}\right),
$$

where $\widehat{H}_{\bar{k}, i}=\left[\widetilde{H}_{1, i}^{H}, \ldots, \widetilde{H}_{\bar{k}, i}^{H}, \ldots, \widetilde{H}_{K_{\Omega}, i}^{H}\right]$ denotes the aggregated interfering channel matrix. Suppose that $\bar{G}_{k, i}$ is the right unitary matrix which can be obtained by the SVD of $\left(\sigma_{n}^{2} I_{\xi+1}+\right.$ $\left.\widehat{H}_{\bar{k}, i}^{H} \widehat{H}_{\bar{k}, i}\right)^{-1}$ and $G_{k, i}$ can be gotten by taking the first column of $\bar{G}_{k, i}$.

From the above descriptions, although the derived precoding matrices cannot nullify the generated interference thoroughly, the OCI suffered by each user can be effectively mitigated by minimizing the aggregated LN. Thus, the SINR of every user rather than that of $\xi$ users can be improved. Moreover, since the proposed method designs the precoder decentralized with partial CSI and no sharing among neighboring $\mathrm{BSs}$, it is very easy to be implemented in real networks.

\section{Decentralized User Selection with Interference Assignment (DUSIA)}

In the above analysis, in order to ensure that the null space of $\bar{H}_{k, i}$ is not empty, the sum of the supported users and $\xi$ is assumed to be less than the transmit antennas at each BS. However, since the number of users in a cell is always much larger than that of antennas implemented at each BS, the BS cannot support all users in one transmit time interval (TTI). When round robin (RR) scheduling is adopted with $\bar{K}(\bar{K} \geq N)$ users in each cell, it needs $\lceil\bar{K} /(N-\xi)\rceil$ TTIs to provide service to all of users. In this case, we need to determine the optimal user subset for each TTI. The problem of sum capacity maximization for each TTI can be formulated as

$$
\begin{gathered}
\max \sum_{i} \sum_{k=1}^{\bar{K}_{i}(t)} C_{k, i}(t), \\
\sum_{k=1}^{\bar{K}_{i}(t)} S_{k, i}+\xi \leq N, \quad S_{k, i}=\{0,1\}, \\
S_{k, i}= \begin{cases}0, & \text { if user } k \text { is not selected, } \\
1, & \text { if user } k \text { is selected. }\end{cases}
\end{gathered}
$$

In (12), $\bar{K}_{i}(t)$ is the number of candidate users for $t$ th TTI. $C_{k, i}(t)$ denotes the user's achievable capacity for $t$ th TTI seen by user $k$ in cell $i$ and can be denoted as

$$
C_{k, i}(t)=\log _{2}\left|I+H_{k, i} W_{k, i} W_{k, i}^{H} H_{k, i}^{H}\left(Z_{k, i}\right)^{-1}\right|,
$$

where $z_{k, i}=\sum_{j \neq i}^{J} \sum_{\bar{k}=1}^{N-\xi} H_{k, j} W_{\bar{k}, j} x_{\bar{k}, j}+n_{k, i}$, and it denotes the sum of the noise and OCI from other BSs. The covariance of $z_{k, i}$ is

$$
Z_{k, i}=E\left\{z_{k, i} z_{k, i}^{H}\right\}=\sum_{j \neq i}^{J} \sum_{\bar{k}=1}^{N-\xi} H_{k, j} W_{\bar{k}, j} W_{\bar{k}, j}^{H} H_{k, j}^{H}+\sigma_{n}^{2} .
$$


According to (13) and (14), we need the explicit information of interfering channels and precoding matrices of users served by adjacent BSs to calculate the user's achievable capacity. In order to solve the problem formulated in (12), global optimal user selection with full CSI sharing and centralized processing is needed to maximize the sum capacity. However, since information sharing that brings large amount of signaling overhead is impractical to be realized via the backhaul links with limited capacity, decentralized user selection with partial CSI sharing for multicell MU-MIMO systems is investigated.

In order to solve the problem described in (12) with a distributed method, the lower bound on the capacity is derived based on the eigenvalues of interfering channels at first. And then, the user subset with maximum sum capacity is selected with the decentralized algorithm named as DUSIA.

4.1. Eigenvalue-Based Lower Bound on the Capacity. For a given TTI, the TTI index in (13) can be ignored for simplification. Thus, the achievable capacity $C_{k, i}(t)$ in (13) seen by user $k$ in cell $i$ can be written as

$$
\begin{aligned}
C_{k, i} & =\log _{2}\left|I+H_{k, i} W_{k, i} W_{k, i}^{H} H_{k, i}^{H}\left(Z_{k, i}\right)^{-1}\right| \\
& >\log _{2}\left|H_{k, i} W_{k, i} W_{k, i}^{H} H_{k, i}^{H}\left(Z_{k, i}\right)^{-1}\right| .
\end{aligned}
$$

The bound in (15) is tight in the high SNR region and less tight in low SNR region. According to $|A B|=|A||B|$ and $\left|A^{-1}\right|=$ $|A|^{-1}$, (15) can be denoted as

$$
C_{k, i}>\log _{2}\left(\left|H_{k, i} W_{k, i} W_{k, i}^{H} H_{k, i}^{H}\right|\left|Z_{k, i}\right|^{-1}\right)
$$

With the definition of $Z_{k, i}$ in (14), $\left|Z_{k, i}\right|$ can be expressed as

$$
\begin{aligned}
\left|Z_{k, i}\right| & =\left|\sum_{j \neq i}^{J} \sum_{\bar{k}=1}^{N-\xi} H_{k, j} W_{\bar{k}, j} W_{\bar{k}, j}^{H} H_{k, j}^{H}+\sigma_{n}^{2}\right| \\
& =\lambda_{1}\left(Z_{k, i}\right) \lambda_{2}\left(Z_{k, i}\right) \cdots \lambda_{s}\left(Z_{k, i}\right) \cdots \lambda_{S}\left(Z_{k, i}\right) .
\end{aligned}
$$

In (17), $\lambda_{s}\left(Z_{k, i}\right)$ is the $s$ th eigenvalue of $Z_{k, i}$. Since $Z_{k, i}$ is a scalar, $\left|Z_{k, i}\right|$ is equal to $\lambda_{1}\left(Z_{k, i}\right)$ which is the maximum eigenvalue of matrix $Z_{k, i}$. For a given user $k$, according to Weyl's theorem $\lambda_{s}(A+B) \leq \lambda_{s}(A)+\lambda_{1}(B)[32], \lambda_{1}\left(Z_{k, i}\right)$ can be expressed as

$$
\lambda_{1}\left(Z_{k, i}\right) \leq \lambda_{1}\left(\sigma_{n}^{2}\right)+\lambda_{1}\left(\widehat{Z}_{k, i}\right)
$$

where $\widehat{Z}_{k, i}=\sum_{j \neq i}^{J} \sum_{\bar{k}=1}^{N-\xi} H_{k, j} W_{\bar{k}, j} W_{\bar{k}, j}^{H} H_{k, j}^{H}$. Since $\lambda_{1}\left(\sigma_{n}^{2}\right)=\sigma_{n}^{2}$, (18) can be rewritten as

$$
\lambda_{1}\left(Z_{k, i}\right) \leq \sigma_{n}^{2}+\lambda_{1}\left(\widehat{Z}_{k, i}\right) .
$$

For arbitrary $k$, since $H_{k, j} W_{\bar{k}, j} W_{\bar{k}, j}^{H} H_{k, j}^{H}$ is the nonnegative scalar, $\lambda_{1}\left(\widehat{Z}_{k, i}\right)$ can be gotten as

$$
\begin{aligned}
\lambda_{1}\left(\widehat{Z}_{k, i}\right) \leq & \lambda_{1}\left(H_{k, 1} W_{1,1} W_{1,1}^{H} H_{k, 1}^{H}\right)+\lambda_{1}\left(H_{k, 1} W_{2,1} W_{2,1}^{H} H_{k, 1}^{H}\right) \\
& +\cdots+\lambda_{1}\left(H_{k, j} W_{\bar{k}, j} W_{\bar{k}, j}^{H} H_{k, j}^{H}\right) \\
& +\cdots+\lambda_{1}\left(H_{k, J} W_{N-\xi, J} W_{N-\xi, J}^{H} H_{k, J}^{H}\right) \\
= & \widetilde{\sigma}_{1}^{2}\left(H_{k, 1} W_{1,1}\right)+\widetilde{\sigma}_{1}^{2}\left(H_{k, 1} W_{2,1}\right) \\
& +\cdots+\widetilde{\sigma}_{1}^{2}\left(H_{k, j} W_{\bar{k}, j}\right)+\cdots+\widetilde{\sigma}_{1}^{2}\left(H_{k, J} W_{N-\xi, J}\right) \\
= & \sum_{j \neq i}^{J} \sum_{\bar{k}=1}^{N-\xi} \widetilde{\sigma}_{1}^{2}\left(H_{k, j} W_{\bar{k}, j}\right),
\end{aligned}
$$

where $\widetilde{\sigma}_{1}\left(H_{k, i} W_{\bar{k}, j}\right)$ is the maximum singular value of matrix $H_{k, i} W_{\bar{k}, j}$. Because $W_{\bar{k}, j}=V_{\bar{k}, j}^{(0)} G_{\bar{k}, j}$ and $G_{\bar{k}, j}$ is one of the columns of unitary matrix $\bar{G}_{\bar{k}, j}$ that can be achieved by the SVD of $\left(\sigma_{n}^{2} I_{\xi+1}+\widehat{H}_{\bar{k}, i}^{H} \widehat{H}_{\bar{k}, i}\right)^{-1}, H_{k, j} W_{\bar{k}, j}$ can be obtained by deleting some columns of matrix $H_{k, j} V_{\bar{k}, j}^{(0)} \bar{G}_{\bar{k}, j}$. According to the singular value inequalities [33], since $\bar{G}_{\bar{k}, j}$ is unitary matrix that cannot change the singular value of a matrix, we can get $\widetilde{\sigma}_{1}\left(H_{k, j} W_{\bar{k}, j}\right)$ as $\tilde{\sigma}_{1}^{2}\left(H_{k, j} W_{\bar{k}_{, j}}\right) \leq \tilde{\sigma}_{1}^{2}\left(H_{k, j} V_{\bar{k}, j}^{(0)} \bar{G}_{\bar{k}, j}\right)=$ $\tilde{\sigma}_{1}^{2}\left(H_{k, j} V_{\bar{k}, j}^{(0)}\right)$. Moreover, since $V_{\bar{k}, j}^{(0)}$ is composed of columns of unitary matrix $V_{\bar{k}, j}, H_{k, j} V_{\bar{k}, j}^{(0)}$ can be derived by deleting some columns of matrix $H_{k, j} V_{\bar{k}, j}$, and $\tilde{\sigma}_{1}^{2}\left(H_{k, j} V_{\bar{k}, j}^{(0)}\right) \leq$ $\widetilde{\sigma}_{1}^{2}\left(H_{k, j} V_{\bar{k}, j}\right)=\widetilde{\sigma}_{1}^{2}\left(H_{k, j}\right)$. Therefore, (20) can be expressed as

$$
\begin{aligned}
\lambda_{1}\left(\widehat{Z}_{k, i}\right) \leq & \widetilde{\sigma}_{1}^{2}\left(H_{k, 1}\right)+\widetilde{\sigma}_{1}^{2}\left(H_{k, 1}\right)+\cdots+\widetilde{\sigma}_{1}^{2}\left(H_{k, j}\right) \\
& +\cdots+\widetilde{\sigma}_{1}^{2}\left(H_{k, J}\right)=\sum_{j \neq i}^{J} K_{j} \widetilde{\sigma}_{1}^{2}\left(H_{k, j}\right) .
\end{aligned}
$$

In (21), $K_{j}$ denotes the number of selected users served by BS $j$, and $K_{j}=N-\xi$ commonly. According to (18)-(21), (17) can be obtained as

$$
\left|Z_{k, i}\right| \leq \sigma_{n}^{2}+\lambda_{1}\left(\widehat{Z}_{k, i}\right) \leq \sigma_{n}^{2}+\sum_{j \neq i}^{J} K_{j} \widetilde{\sigma}_{1}^{2}\left(H_{k, j}\right) .
$$

With (22), (16) can be rewritten as

$$
C_{k, i}>\log _{2} \frac{\left|H_{k, i} W_{k, i} W_{k, i}^{H} H_{k, i}^{H}\right|}{\sigma_{n}^{2}+\sum_{j \neq i}^{J} K_{j} \lambda_{1}\left(H_{k, j} H_{k, j}^{H}\right)} .
$$

Thus, the eigenvalue-based lower bound on the capacity can be derived as

$$
\bar{C}_{k, i}=\log _{2} \frac{\left|H_{k, i} W_{k, i} W_{k, i}^{H} H_{k, i}^{H}\right|}{\sigma_{n}^{2}+\sum_{j \neq i}^{J} K_{j} \lambda_{1}\left(H_{k, j} H_{k, j}^{H}\right)} .
$$


When $K_{j}=0$, the lower bound capacity with othercell interference expressed in (24) becomes the capacity without OCI. From (24), we can see that the lower bound on the capacity is affected by the maximum eigenvalue of interfering channels and the number of selected users served by interfering BSs merely.

4.2. User Selection with Lower Bound on the Capacity. According to (24), the lower bound on the capacity is independent of explicit information of interfering channels and precoding matrices of users served by adjacent BSs. When we replace the $C_{k, i}(t)$ in (10) with the lower bound on the capacity $\bar{C}_{k, i}(t)$, the result of user selection for each adjacent cell will not be affected mutually. Thus, the problem formulated in (10) can be reformulated to the problem of maximizing the sum capacity for a single cell, and it can be expressed as

$$
\begin{gathered}
\max \sum_{k=1}^{\bar{K}_{i}(t)} \bar{C}_{k, i}(t) \\
\sum_{k=1}^{\bar{K}_{i}(t)} S_{k, i}+\xi \leq N, \quad S_{k, i}=\{0,1\}, \\
S_{k, i}= \begin{cases}0, & \text { if user } k \text { is not selected, } \\
1, & \text { if user } k \text { is selected. }\end{cases}
\end{gathered}
$$

Generally, exhaustive search can be used to find the optimal user subset, but this method will bring prohibitive computation complexity when user scale becomes large. Hence, a suboptimal greedy user selection algorithm for each TTI is proposed with lower bound on the capacity. In the process of DUSIA, a single user is added every time till $N-\xi$ users have been selected to provide maximum sum capacity. Let $\bar{C}_{k, i}(t)$ represent the instantaneous capacity when the user $k$ is selected. $(k, i)$ denotes that the user $k$ served by BS $i$ is selected.

In stage $n=1$ of DUSIA, since the selected user set $\Omega_{i}(t)=\emptyset$, the Frobenius norm of $H_{k, i}$ is used to get the lower bound of sum capacity while, in other stages, the precoding matrix $W$ is obtained from the proposed precoding method and (24) is used to calculate the lower bound on the sum capacity. Meanwhile, the BS keeps adding one user at a time till the number of active users reaches $N-\xi$. Furthermore, since DUSIA only uses the eigenvalues of interfering channels, it does not need the full CSI and precoding matrices sharing during the user selection.

\section{Performance Comparison and Analysis}

In this section, $\mathrm{BD}$ with capacity-based user selection (BDCUS) in [34] and interference nulling with user selection (INUS) in [30] are selected as reference schemes. For INUS, zero-force beamforming (ZFBF) is used to design the precoding matrices for each user, and the generated interference to other cell $\xi$ users is nullified by sacrificing $\xi$ degrees of freedom. Moreover, the effects of the number of antennas

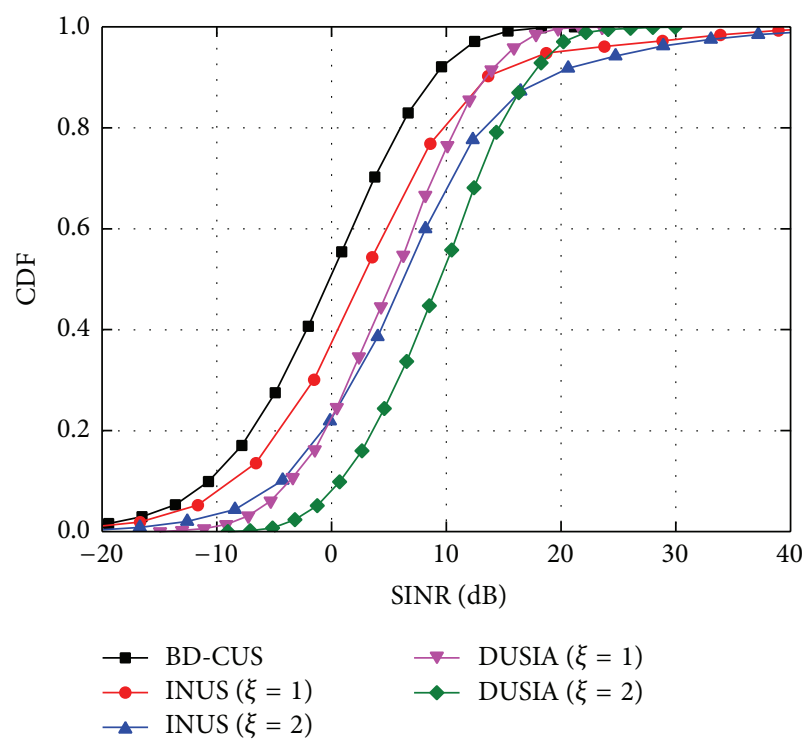

FIGURE 2: CDF of users' achievable SINR comparison with $N=4$ and 10 users per cell.

implemented at each BS, interference-free SNR at the cell edge, and degrees of freedom used to mitigate generated interference are also investigated with DUSIA.

In the simulation, a multicell downlink MU-MIMO system consisting of seven transmitting BSs with same frequency is assumed. Each BS uses 120-degree sectoring antenna to create 3 cells. The numbers of antennas at each BS and user are set to be $N$ and 1 , respectively. The users are uniformly distributed in one cell. RR is adopted across TTIs to keep the fairness among users. The distance based path loss is $37.6 \log (d)+128.1$, the standard deviation of shadowing is $8 \mathrm{~dB}$, and the cell radius is $1 \mathrm{Km}$. The interference-free SNR at the cell edge, named as cell edge SNR for short, includes the effects of path loss and shadowing. Furthermore, Rayleigh fading is assumed for the channel between the user and the BS, and each element of the channel matrix is i.i.d. with zero mean and unit variance.

5.1. CDF of SINR for Different Precoding Schemes. In Figure 2, the CDF of users' achievable SINR is plotted for different precoding schemes with 10 users uniformly dropped in each cell. Each BS has 4 antennas, and the cell edge SNR is adjusted as $10 \mathrm{~dB} . \xi=1$ and $\xi=2$ are both assumed to show the characteristics of INUS and DUSIA, respectively. As shown in Figure 2, compared with BD-CUS, the larger $\xi$ is, the more SINR gain can be achieved by DUSIA. In addition, sacrificing $\xi$ degrees of freedom to mitigate OCI, either by nullifying generated interference or minimizing generated interference, is always helpful in terms of SINR distribution. For INUS, since it can nullify the generated interference to $\xi$ users, it can only improve the SINR of the $\xi$ users without fairness among users. While for DUSIA, since it can distribute the benefits achieved by sacrificing degrees of freedom to all of users instead of $\xi$ users, every user's SINR can be improved by minimizing the aggregated generated interference for all 


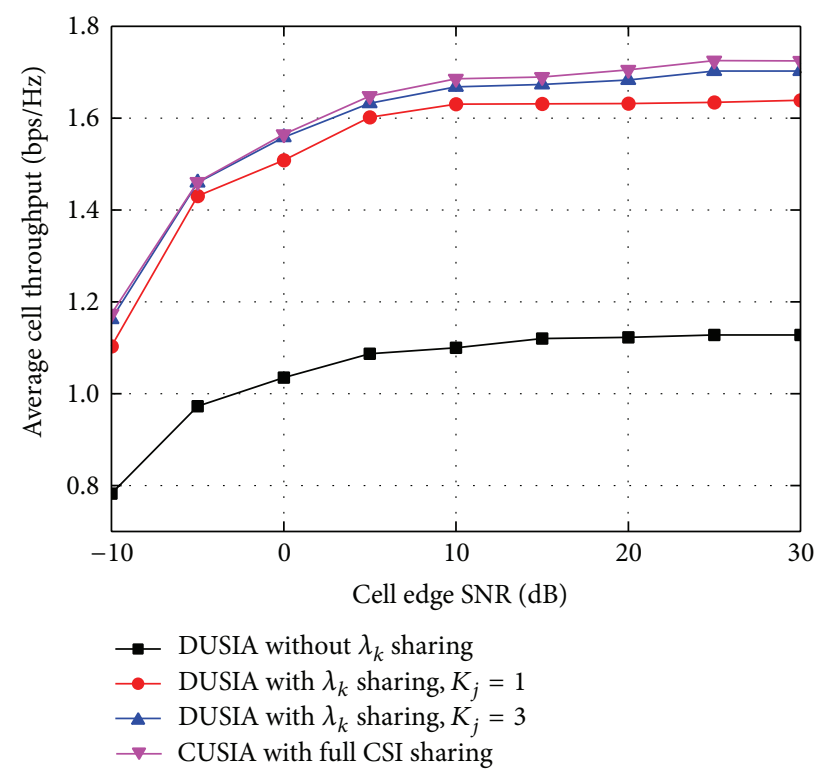

FIgURE 3: Cell throughput comparison of DUSIA with different level of information sharing, $N=4, \xi=1,10$ users per cell.

other cell users, so the $5 \%$ CDF of SINR of DUSIA is larger than that of INUS. For example, with $\xi=1$, DUSIA improves the $5 \%$ CDF of SINR of INUS from $-12 \mathrm{~dB}$ to $-7 \mathrm{~dB}$.

\subsection{Comparison of DUSIA with Different Level of Information}

Sharing. Figure 3 shows the average cell throughput as a function of cell edge SNR for DUSIA with different level of information sharing and $N=4, \xi=1$. For Coordinated User Selection based on Interference Assignment (CUSIA) with full CSI sharing, we assume that the full CSI of interfering channels and precoding information of other cell users can be shared among BSs ideally, and multiple BSs select their serving users coordinately. Suppose $\lambda_{k}$ is the eigenvalue of interfering channels for user $k$, for DUSIA without $\lambda_{k}$ sharing, $\lambda_{k}$ is set as 0 , and the user selection process in DUSIA becomes the capacity-based user selection without considering the OCI. Compared with DUSIA without $\lambda_{k}$ sharing, DUSIA with $\lambda_{k}$ sharing can improve the average cell throughput more than $50 \%$. In addition, the accuracy of $K_{j}$ can also affect the performance of DUSIA with $\lambda_{k}$ sharing. In the simulation, since three users can be selected for one TTI with $N=4, \xi=1$, the average cell throughput of DUSIA with $K_{j}=3$ outperforms $K_{j}=1$ by $5 \%$. At last, since the eigenvalues of interfering channels are shared to assist in selecting users, the throughput losses of DUSIA are marginal compared to CUSIA that overloaded with signaling overhead. As shown in Figure 3, when the cell edge SNR is below $0 \mathrm{~dB}$, the performance of DUSIA and CUSIA with full CSI sharing is almost the same. Even when the cell edge SNR is $30 \mathrm{~dB}$, DUSIA can still achieve more than $99 \%$ cell throughput of CUSIA with full CSI sharing.

5.3. Throughput Gain with $\xi=1$. The average cell throughput and cell edge throughput as a function of cell edge SNR are

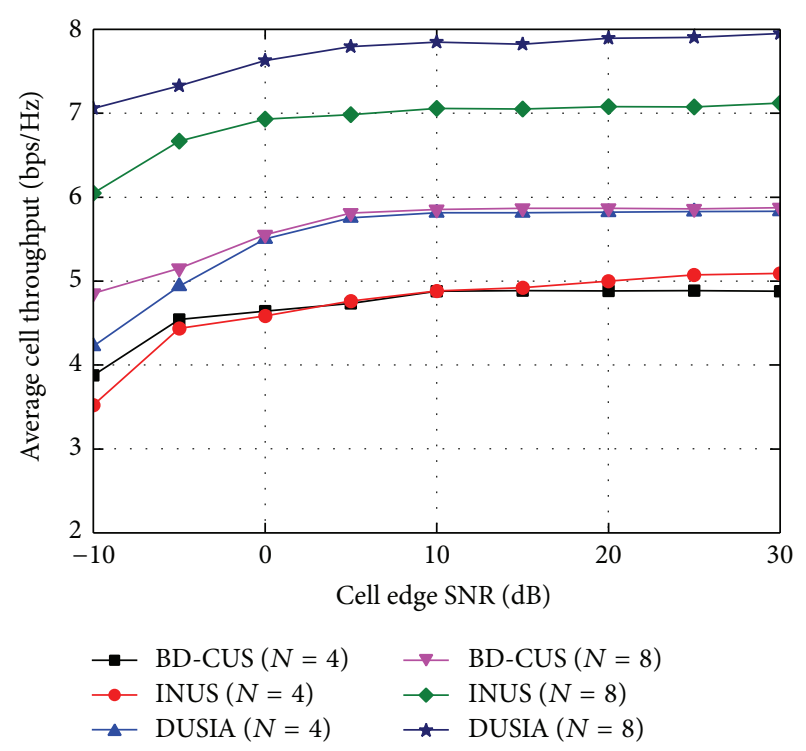

FIGURE 4: Average cell throughput comparison of BD-CUS, INUS, and DUSIA. $\xi=1,10$ users per cell.

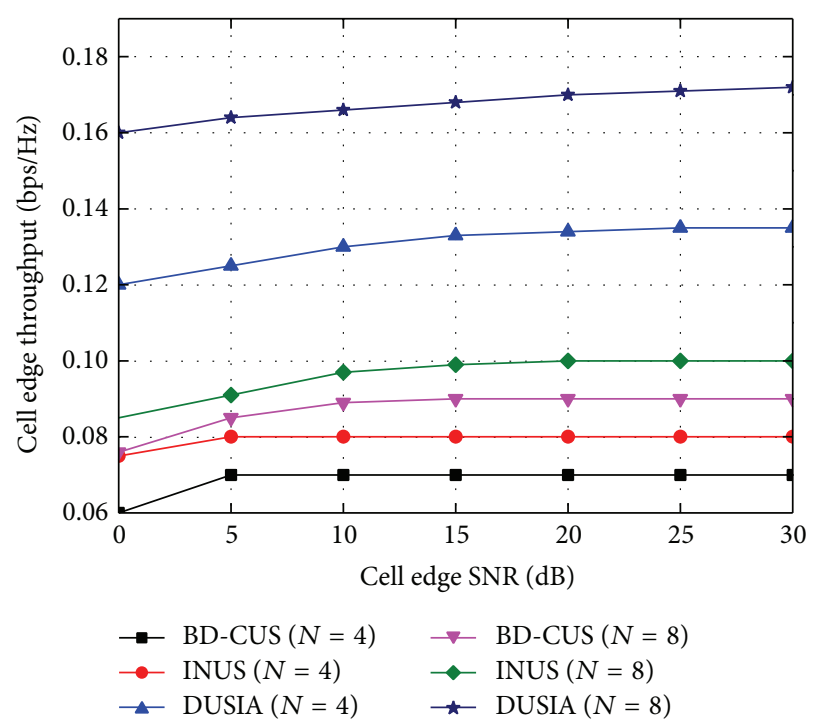

FIGURE 5: Cell edge throughput comparison of BD-CUS, INUS, and DUSIA. $\xi=1,10$ users per cell.

plotted with $\xi=1$ and 10 users per cell in Figures 4 and 5, respectively. In each figure, the performances of $N=4$ and 8 are both investigated.

As shown in Figure 5, compared with INUS of $N=4$ in terms of cell edge throughput, although DUSIA cannot nullify the generated interference for $\xi$ users, it can increase the SINR of every user rather than that of $\xi$ users by minimizing the aggregated generated interference for other cell users. Thus, DUSIA can increase the cell edge throughput of INUS up to $70 \%$. This suggests that minimizing the aggregated generated interference for all of other cell users is more favorable than nullifying generated interference in terms of the cell edge throughput. 
Besides the cell edge throughput gain, DUSIA with $\xi=1$ can also achieve performance gain in terms of average cell throughput regardless of $N$ and cell edge SNR as shown in Figure 4. Compared with INUS of $N=4$, although the maximum achievable SINR of DUSIA is lower than that of INUS as shown in Figure 2, DUSIA can still increase the average cell throughput of INUS up to $20 \%$. This is because the medium SINR corresponding to $50 \%$ CDF of DUSIA is $2 \mathrm{~dB}$ higher than that of IUAS and the sum capacity for each TTI is maximized by the eigenvalue-based user selection scheme with the effects of interfering channels being considered.

What is more important, the average cell throughput gain of DUSIA is changeable along with the $N$ and cell edge SNR. For example, with $N=4$ and the cell edge SNR being $-10 \mathrm{~dB}$ in Figure 4, the average cell throughput gain of DUSIA compared with BD-CUS is about $0.3 \mathrm{bps} / \mathrm{Hz}$. While for $N=4$ and cell edge SNR being $30 \mathrm{~dB}$, the average cell throughput gain is about $1 \mathrm{bps} / \mathrm{Hz}$. In addition, for $N=8$ and cell edge SNR being $-10 \mathrm{~dB}$, the average cell throughput gain is about $2 \mathrm{bps} / \mathrm{Hz}$. This changeable throughput gain suggests that the throughput is affected by several factors, such as the number of antennas implemented at each BS and the cell edge SNR.

5.4. Throughput Analysis for Best Choice of $\xi$. Figures 6 and 7 show the cell throughput and CDF of user throughput for DUSIA with different $(N, \xi)$ respectively. As shown in Figure 6 with $(4,1)$, DUSIA can increase the average cell throughput from $2.3 \mathrm{bps} / \mathrm{Hz}$ to $4 \mathrm{bps} / \mathrm{Hz}$ along with the raise of the cell edge SNR from $-10 \mathrm{~dB}$ to $5 \mathrm{~dB}$. This suggests the DUSIA is more favorable in the high level OCI scenarios, since the noise rather than the OCI is the dominant factor that affects the average cell throughput in the low level OCI scenarios. In addition, compared with the performance of DUSIA with $N=4$, DUSIA with $N=8$ can achieve better performance both in terms of average cell throughput and cell edge throughput, since 8 antennas implemented at each BS can bring more beamforming gain than 4 antennas. For example, with cell edge SNR being $20 \mathrm{~dB}$ in Figures 6 and 7 , DUSIA with $(8,2)$ can increase the cell edge throughput and average cell throughput of DUSIA with $(4,2)$ up to $90 \%$ and $82 \%$, respectively.

Besides, although fewer active users can be supported with larger $\xi$, the cell edge throughput defined as the $5 \% \mathrm{CDF}$ of user throughput can still be improved with the increase of $\xi$, since every user can get more SINR gain by using larger $\xi$ to minimize the generated interference which is proven in Figure 2. For example, with $N=4$ shown in Figure 7, the cell edge throughput for $\xi=1$ is about $0.12 \mathrm{bps} / \mathrm{Hz}$. While for $\xi=2$ and $\xi=3$, the cell edge throughput can be achieved is about $0.13 \mathrm{bps} / \mathrm{Hz}$ and $0.15 \mathrm{bps} / \mathrm{Hz}$, respectively. This suggests that using $\xi$ degrees of freedom, regardless of the value of $\xi$, to minimize the generated interference is always helpful for cell edge users who bear high OCI. And the larger the $\xi$ is, the more cell edge throughput can be achieved. However, the bonus for cell edge throughput cannot be realized for average cell throughput. For example, with $N=4$, although DUSIA with $(4,3)$ has the largest cell edge throughput, its average cell

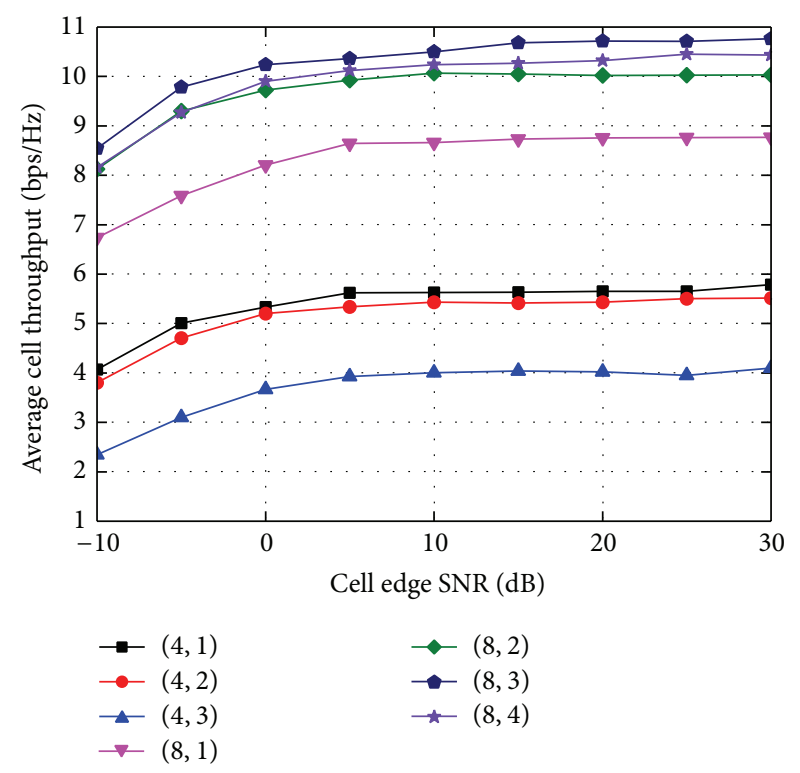

FIGURE 6: Cell throughput for DUSIA with different $(N, \xi)$ versus cell edge SNR.

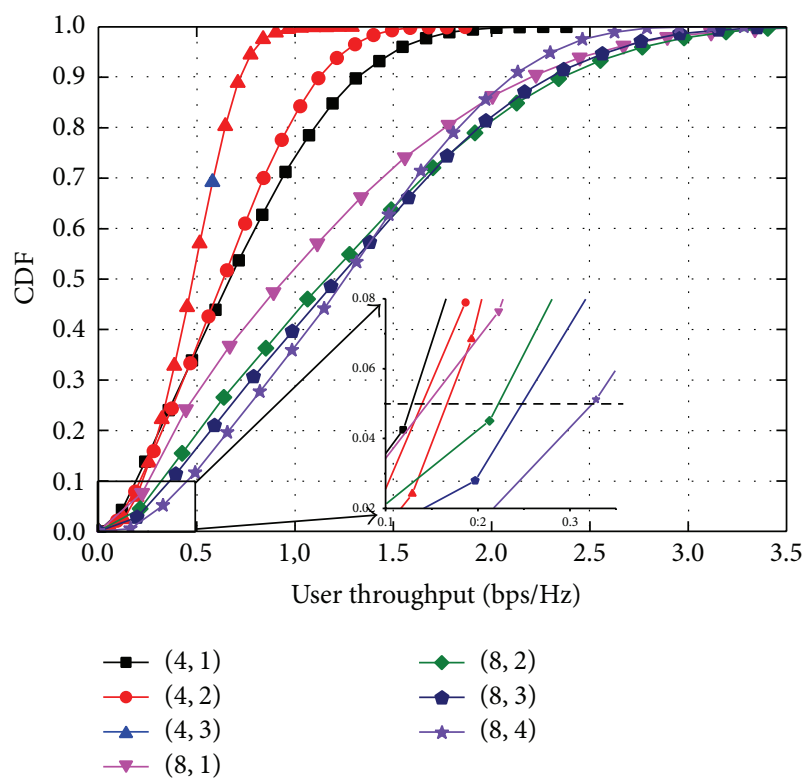

FIGURE 7: CDF of user throughput for DUSIA with different $(N, \xi)$. Cell edge $\mathrm{SNR}=20 \mathrm{~dB}$.

throughput is far below the others as shown in Figure 6, since it can only support a single user during one TTI.

Furthermore, although DUSIA with $\xi=1$ can achieve best performance in terms of average cell throughput with $N=4$, it is not mean that $\xi=1$ is the best choice in terms of average cell throughput for all of scenarios. As shown in Figure 6 with $N=8$, the best performance in terms of average cell throughput can be achieved with $\xi=3$. Meanwhile, the performance of DUSIA with $(8,4)$ is better than that of DUSIA with $(8,1)$ and $(8,2)$. This suggests that the best choice of $\xi$ for average cell throughput varies with the number of antennas implemented at each BS. 


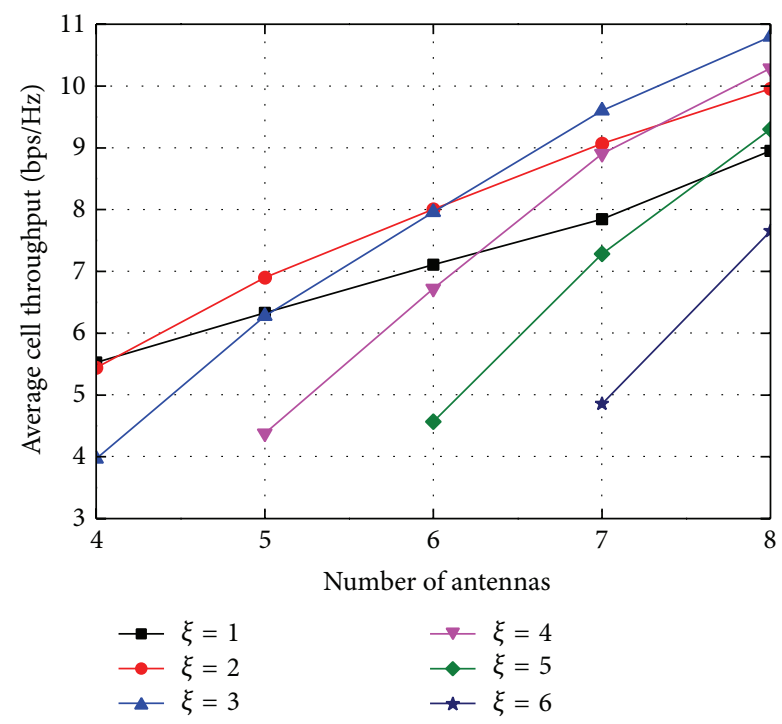

FIGURE 8: Cell throughput for DUSIA with different $\xi$ versus number of antennas. Cell edge SNR $=20 \mathrm{~dB}$.

Figure 8 investigates the average cell throughput with different $\xi$ as a function of number of antennas with the cell edge SNR being $20 \mathrm{~dB}$. As shown in Figure 8, in terms of average cell throughput, the best choice of $\xi$ for every case of antenna configuration is different. For example, with $N=5$, the best choice of $\xi$ is $\xi=2$. While for $N=8, \xi=3$ becomes the best choice. Moreover, for a scenario with fixed number of antennas, the relationship between the average cell throughput and $\xi$ is not linear. For example, with $N=6$, the relationship of average cell throughput with different $\xi$ is $\xi=2>\xi=3>\xi=1>\xi=4>\xi=5$.

From Figures 7 and 8, we can see that the best choice of $\xi$ for cell edge throughput is $N-1$. While for average cell throughput, there is a turning point with the increasement of $\xi$, and the value of turning point varies with $N$. Therefore, it is hard to figure out the best choice of $\xi$ for all metrics regardless of the scenarios, such as number of antennas and cell edge SNR. However, we can obtain some suboptimal solutions according to system requirements. For example, DUSIA with $(6,3)$ can be adopted instead of $(6,2)$ to improve the cell edge throughput without serious penalty to average cell throughput.

\section{Conclusion}

In this paper, we propose an altruistic precoding solution for downlink MU-MIMO systems by performing the eigenvalues-based user selection and precoder design. In DUSIA, the level of IUI among users is assigned as zero and the level of OCI generated to other cell users is assigned to minimize the sum of generated interference by using $\xi$ degrees of freedom. Moreover, with the eigenvalue-based lower bound on the capacity being used as a metric to select users, the global optimal problem of user selection can be reformulated to a local optimal problem, which can be tackled by each BS with the eigenvalues of interfering channels being shared merely. Compared with the schemes that nullify the generated interference, DUSIA has better performance both in terms of the average cell throughput and cell edge throughput for any OCI levels.

In addition, the best choice of $\xi$ is investigated with different OCI levels and number of antennas through simulation. The simulation results demonstrate that (1) DUSIA is more favorable at any OCI levels. (2) Given the fixed number of antennas at each BS, the larger the $\xi(1 \leq \xi \leq N-1)$ can be, the more cell edge throughput can be achieved. (3) In terms of average cell throughput, there is a turning point with the increasement of $\xi$, and the value of the turning point varies with the number of antennas implemented at BS.

\section{Conflict of Interests}

The authors declare that there is no conflict of interests regarding the publication of this paper.

\section{Acknowledgment}

This work was supported by the National S\&T Major Project (2010ZX03002-008-01).

\section{References}

[1] R. S. Blum, "MIMO capacity with interference," IEEE Journal on Selected Areas in Communications, vol. 21, no. 5, pp. 793-801, 2003.

[2] M. H. M. Costa, "Writing on dirty paper," IEEE Transactions on Information Theory, vol. 29, no. 3, pp. 439-441, 1983.

[3] Q.H. Spencer, A. L. Swindlehurst, and M. Haardt, “Zero-forcing methods for downlink spatial multiplexing in multiuser MIMO channels," IEEE Transactions on Signal Processing, vol. 52, no. 2, pp. 461-471, 2004.

[4] J. G. Andrews, W. Choi, and R. W. Heath Jr., "Overcoming interference in spatial multiplexing mimo cellular networks," IEEE Wireless Communications, vol. 14, no. 6, pp. 95-104, 2007.

[5] "Further advancements for E-UTRA physical layer aspects," 3GPP Technical Report TR 36.814 V9.0.0 (Release 9), 2010, http://www.3gpp.org.

[6] G. Caire and S. Shamai, "On the achievable throughput of a multiantenna Gaussian broadcast channel," IEEE Transactions on Information Theory, vol. 49, no. 7, pp. 1691-1706, 2003.

[7] H. Zhang and H. Dai, "Cochannel interference mitigation and cooperative processing in downlink multicell multiuser MIMO networks," EURASIP Journal on Wireless Communications and Networking, vol. 2004, no. 2, pp. 222-235, 2004.

[8] M. K. Karakayali, G. J. Foschini, R. A. Valenzuelat, and R. D. Yates, "On the maximum common rate achievable in a coordinated network," in Proceedings of the IEEE International Conference on Communications (ICC '06), pp. 4333-4338, Istanbul, Turkey, July 2006.

[9] R. Zhang, "Cooperative multi-cell block diagonalization with per-base-station power constraints," IEEE Journal on Selected Areas in Communications, vol. 28, no. 9, pp. 1435-1445, 2010.

[10] R. Zakhour and D. Gesbert, "Optimized data sharing in multicell MIMO with finite backhaul capacity," IEEE Transactions on Signal Processing, vol. 59, no. 12, pp. 6102-6111, 2011. 
[11] P. Marsch and G. Fettweis, "On base station cooperation schemes for downlink network MIMO under a constrained backhaul," in Proceedings of the IEEE Global Telecommunications Conference (GLOBECOM '08), pp. 1219-1224, New Orleans, La, USA, December 2008.

[12] J. Zhang, M. Kountouris, J. G. Andrews, and R. W. Heath Jr., "Multi-mode transmission for the MIMO broadcast channel with imperfect channel state information," IEEE Transactions on Communications, vol. 59, no. 3, pp. 803-814, 2011.

[13] J. Xu and L. Qiu, "Robust multimode selection in the downlink multiuser MIMO channels with delayed CSIT," in Proceedings of the IEEE International Conference on Communications (ICC '11), Kyoto, Japan, June 2011.

[14] F. Boccardi and H. Huang, "Limited downlink network coordination in cellular networks," in Proceedings of the 18th Annual IEEE International Symposium on Personal, Indoor and Mobile Radio Communications (PIMRC '07), Athens, Greece, September 2007.

[15] J. Zhang, R. Chen, J. G. Andrews, A. Ghosh, and R. W. Heath, "Networked MIMO with clustered linear precoding," IEEE Transactions on Wireless Communications, vol. 8, no. 4, pp.19101921, 2009.

[16] J.-M. Moon and D.-H. Cho, "Efficient cell-clustering algorithm for inter-cluster interference mitigation in network MIMO systems," IEEE Communications Letters, vol. 15, no. 3, pp. 326328, 2011.

[17] C. T. K. Ng and H. Huang, "Linear precoding in cooperative MIMO cellular networks with limited coordination clusters," IEEE Journal on Selected Areas in Communications, vol. 28, no. 9, pp. 1446-1454, 2010.

[18] H. J. Bang and P. Orten, "Scheduling and feedback reduction in cellular networks with coordination clusters," in Proceedings of the IEEE Wireless Communications and Networking Conference (WCNC '11), pp. 1864-1868, Cancun, Mexico, March 2011.

[19] W. Choi and J. G. Andrews, "The capacity gain from intercell scheduling in multi-antenna systems," IEEE Transactions on Wireless Communications, vol. 7, no. 2, pp. 714-725, 2008.

[20] S. Shim, J. S. Kwak, R. W. Heath Jr., and J. G. Andrews, "Block diagonalization for multi-user MIMO with other-cell interference," IEEE Transactions on Wireless Communications, vol. 7, no. 7, pp. 2671-2681, 2008.

[21] H. Dahrouj and W. Yu, "Coordinated beamforming for the multicell multi-antenna wireless system," IEEE Transactions on Wireless Communications, vol. 9, no. 5, pp. 1748-1759, 2010.

[22] Y. Huang, G. Zheng, M. Bengtsson, K.-K. Wong, L. Yang, and B. Ottersten, "Distributed multicell beamforming with limited intercell coordination," IEEE Transactions on Signal Processing, vol. 59, no. 2, pp. 728-738, 2011.

[23] N. Hassanpour, J. E. Smee, J. Hou, and J. B. Soriaga, "Distributed beamforming based on signal-to-caused-interference ratio," in Proceedings of the IEEE 10th International Symposium on Spread Spectrum Techniques and Applications (ISSSTA '08), pp. 405410, Bologna, Italy, August 2008.

[24] R. Zakhour and D. Gesbert, "Coordination on the MISO interference channel using the virtual SINR framework," in Proceedings of International ITG/IEEE Workshop on Smart Antennas, Berlin, Germany, February 2009.

[25] R. Zakhour and D. Gesbert, "Distributed multicell-MISO precoding using the layered virtual SINR framework," IEEE Transactions on Wireless Communications, vol. 9, no. 8, pp. 2444-2448, 2010.
[26] M. Sadek, A. Tarighat, and A. H. Sayed, "A leakage-based precoding scheme for downlink multi-user MIMO channels," IEEE Transactions on Wireless Communications, vol. 6, no. 5, pp. 1711-1721, 2007.

[27] W. W. L. Ho, T. Q. S. Quek, S. Sun, and R. W. Heath Jr., "Decentralized precoding for multicell MIMO downlink," IEEE Transactions on Wireless Communications, vol. 10, no. 6, pp. 1798-1809, 2011.

[28] M. Yoon, M.-S. Kim, and C. Lee, "Decentralized precoding algorithm with weighted SLNR for limitedly coordinated network," IEEE Communications Letters, vol. 16, no. 3, pp. 318-320, 2012.

[29] J. Zhang and J. G. Andrews, "Adaptive spatial intercell interference cancellation in multicell wireless networks," IEEE Journal on Selected Areas in Communications, vol. 28, no. 9, pp. 1455$1468,2010$.

[30] U. Jang, H. Son, J. Park, and S. Lee, "CoMP-CSB for ICI nulling with user selection," IEEE Transactions on Wireless Communications, vol. 10, no. 9, pp. 2982-2993, 2011.

[31] "X2 general aspects and principles," 3GPP Technical Report TR 36.420 V10.2.0 (Release 10), 2011, http://www.3gpp.org.

[32] P. Lancaster and M. Tismenetsky, The Theory of Matrices: With Applications, Academic Press, 1985.

[33] R. A. Horn and C. R. Johnson, Topics in Matrix Analysis, Cambridge University Press, Cambridge, UK, 1991.

[34] R. Chen, Z. Shen, J. G. Andrews, and R. W. Heath Jr., "Multimode transmission for multiuser MIMO systems with block diagonalization," IEEE Transactions on Signal Processing, vol. 56, no. 7, pp. 3294-3302, 2008. 

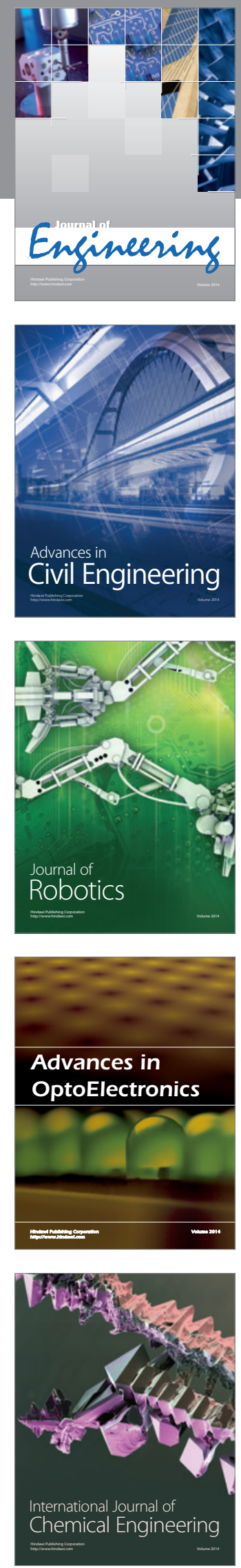

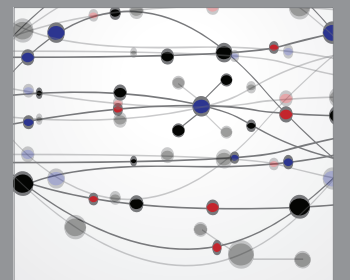

The Scientific World Journal
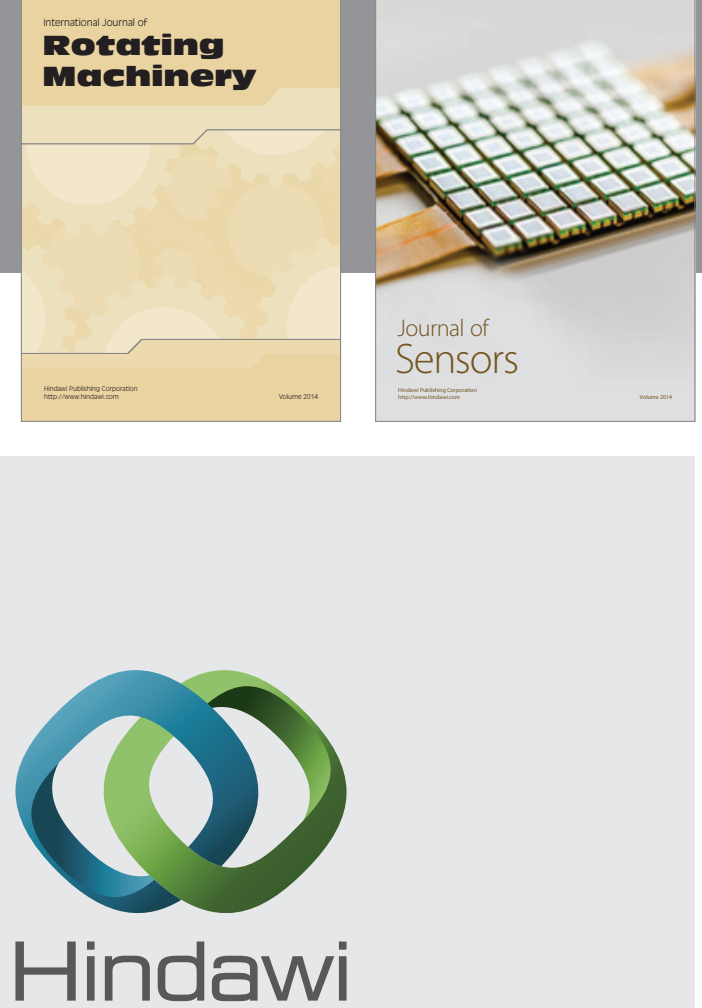

Submit your manuscripts at http://www.hindawi.com
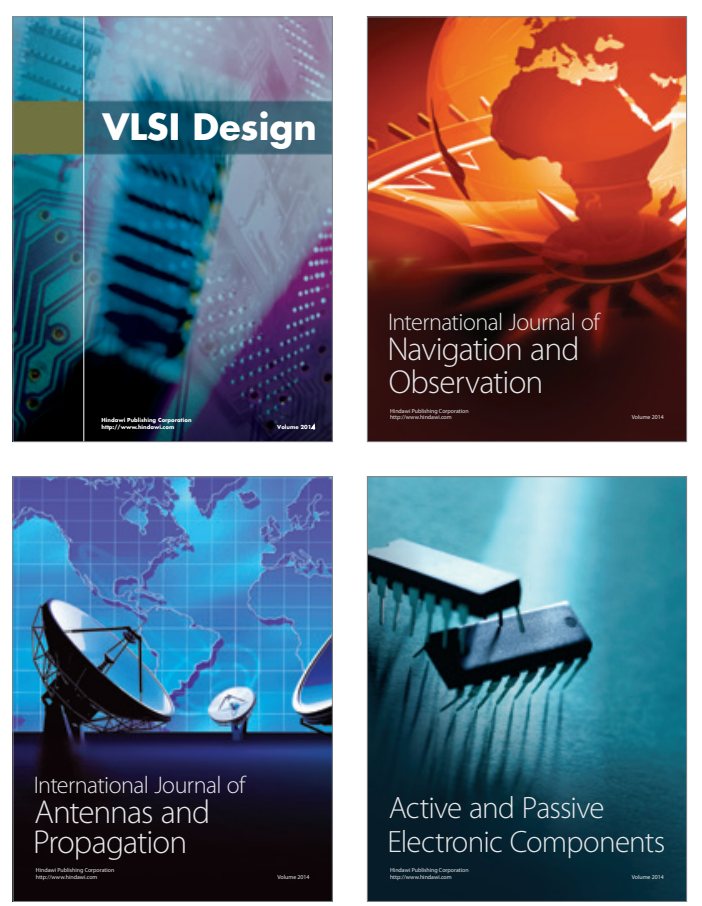
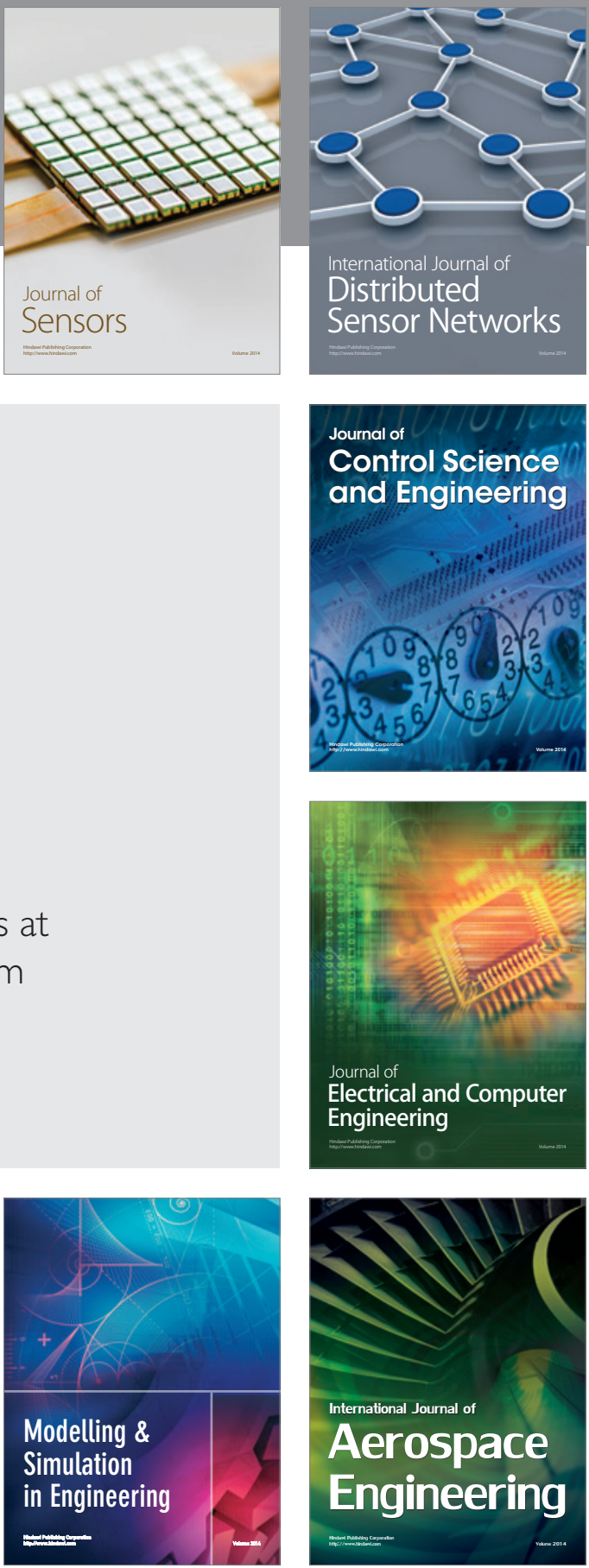

Journal of

Control Science

and Engineering
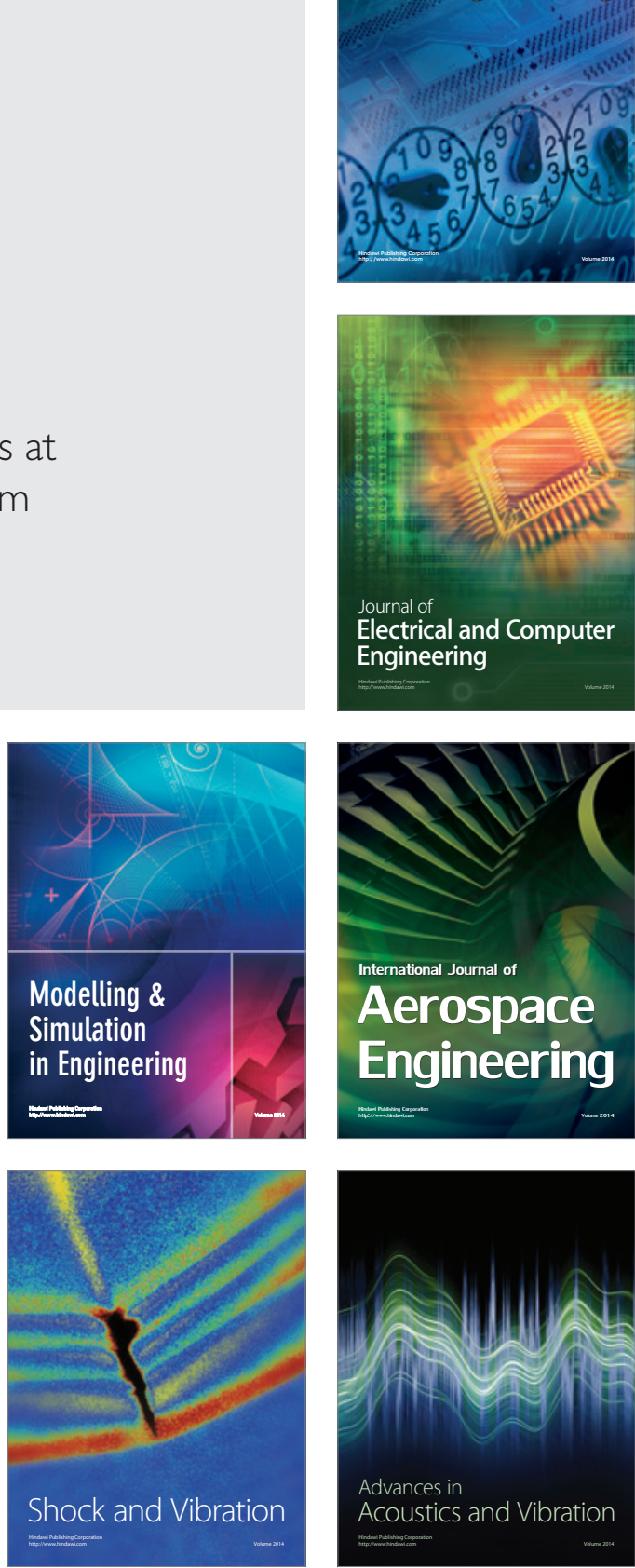